\title{
Modified Development in Transgenic Tobacco Plants Expressing a rolA::GUS Translational Fusion and Subcellular Localization of the Fusion Protein
}

\author{
Françoise Vilaine, ${ }^{1}$ Jacques Rembur, ${ }^{2}$ Dominique Chriqui, ${ }^{2}$ and Mark Tepfer ${ }^{1}$ \\ ${ }^{1}$ Laboratoire de Biologie Cellulaire, INRA-Centre de Versailles, 78026 Versailles Cedex, France; \\ ${ }^{2}$ Laboratoire CEMV, Bât. N2, Université Pierre et Marie Curie, 4, place Jussieu, 75252 Paris Cedex 05, \\ France \\ Accepted 6 June 1998.
}

\begin{abstract}
The rolA gene is transferred naturally by Agrobacterium rhizogenes to the genome of host plants, where it induces dramatic changes in development of transformed plants, including dwarfism and leaf wrinkling. The predicted translation product of the rolA gene is a small $(11.4 \mathrm{kDa})$, basic $(\mathrm{pI}=11.2)$ protein, which has no clearly significant similarity to sequences in the data bases. We have introduced into the tobacco genome a gene encoding a rolA::GUS fusion protein. Expression of this gene led to synthesis of an RNA and a protein of expected size, and the transformed plants exhibited the dwarfism and leaf wrinkling typical of rolA plants, but to a lesser degree than plants transformed with the wild-type rolA gene. The distribution of $\beta$-glucuronidase (GUS) activity was compared in subcellular fractions of leaf extracts from plants expressing either the rolA::gus gene or a control gus construct. As expected, in the control plants, GUS activity was essentially cytosolic. In contrast, in plants expressing the rolA::gus gene the highest specific activity was associated with the plasmalemma fraction.
\end{abstract}

The rol (root locus) genes (White et al. 1985), which are transferred naturally by Agrobacterium rhizogenes from the $\mathrm{Ri}$ plasmid to the nuclear genome of host plants, have profound effects on plant development (Tepfer 1984). When transformed with the rolA gene of strains A4 or 2659, plants of several species display a characteristic set of morphological modifications, including pronounced dwarfism, leaf wrinkling, and reduced fertility (Sinkar et al. 1988; Schmülling et al. 1988; Serino et al. 1994). The function encoded by rolA is at this time unknown. The rolA protein is predicted from the sequence to be of low molecular mass $(11.4 \mathrm{kDa})$ and high isoelectric point (11.2), which has led to the hypothesis that it could interact with nucleic acids, and might be involved in regulation of gene expression (Levesque et al. 1988).

In order to better understand the morphological modifications of rolA plants, certain aspects of the physiology of tobacco plants expressing rolA have been examined. (Martin-

Corresponding author: Mark Tepfer, Laboratoire de Biologie Cellulaire, INRA-Centre de Versailles, Versailles Cedex, France; Telephone: + 331 308330 29; Fax: + 331308330 99; E-mail: mark@ versailles.inra.fr
Tanguy et al. 1990; Maurel et al. 1991; Vansuyt et al. 1992; Dehio et al. 1993; Prinsen et al. 1994; Ben-Hayyim et al. 1996). The physiological studies of rolA plants contribute to our understanding of the cascade of events responsible for the morphological modifications observed in rolA plants, but are of limited use in predicting the nature of the rolA gene product itself. However, determination of the subcellular localization of the rolA protein would constitute a first step in understanding its function, since a DNA-binding protein would be expected to be nuclear, one involved in regulating hormone metabolism would probably be cytosolic, and one involved in hormone sensitivity or in transduction of hormone signals could be expected to be membrane localized. We have introduced into tobacco a gene encoding a bifunctional translational fusion between rolA and the Escherichia coli $\beta$ glucuronidase (GUS), and present here studies of the expression of the fusion gene and the localization of the fusionprotein gene product.

\section{RESULTS}

Construction of the rolA::gus gene and its transfer to the tobacco genome.

The four predicted rolA protein sequences were aligned to facilitate identification of conserved features (Fig. 1). The Nterminal half of the predicted rolA protein is highly conserved among the four strains, whereas the C-terminal half of the A4 rolA protein diverges from the other three. Examination of the A4 rolA nucleotide sequence, as confirmed by two different groups (Slightom et al. 1986; Sinkar et al. 1988), shows that this sequence divergence is due to a frameshift caused by a single nucleotide insertion/deletion in the A4 sequence after the codon of the serine residue at position 64 , while the nucleotide sequence is equally well conserved over the entire rolA coding region (not shown).

The most striking conserved feature of the rolA protein sequences is the highly basic domain (residues 27 to 38). This domain is essential for rolA function, since plants do not display the rolA phenotype when transformed with rolA genes in which mutations affect either the Arg at position 38 or the Pro at position 41 (Dehio and Schell 1993). If the rolA protein is nuclear, then the basic domain could also contribute to its transport to this compartment (Raikhel 1992). 
For two reasons, we chose to construct a gene that would fuse the $\mathrm{C}$ terminus of the rolA peptide to the $\mathrm{N}$ terminus of the GUS protein, rather than the inverse. The above sequence analysis showed that the C-terminal portion of rolA is less conserved, and it has been shown that fusion of certain nuclear localizing signals to the $\mathrm{N}$ terminus of GUS is more efficient for nuclear transport than the corresponding C-terminal fusion (Raikhel 1992). Tobacco was transformed by standard protocols, and individuals expressing the rolA::gus gene were selfed in two successive generations to yield the homozygous plants used in further studies. Plant lines transformed with the rolA::gus gene were designated RAG(n), where $\mathrm{n}$ is the line number.

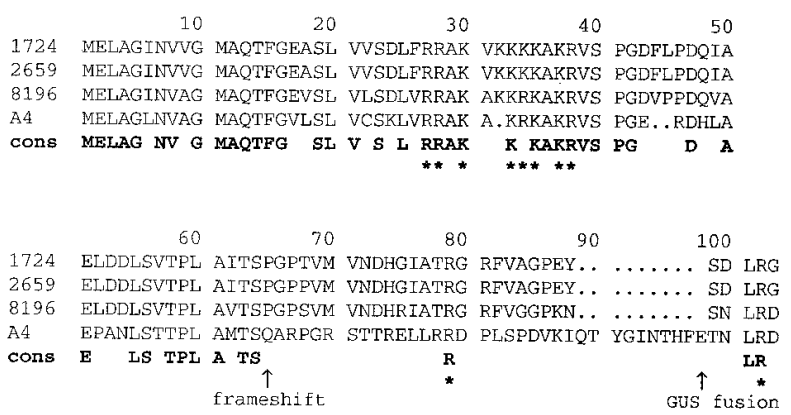

Fig. 1. Comparison of rolA protein sequences. Peptide sequences were deduced from nucleotide sequences of the rolA genes of Agrobacterium rhizogenes strains $1724,2659,8196$, and A4. Below the alignment is shown a consensus sequence (residues identical in all four proteins); positions where basic residues are conserved, indicated with asterisks. Position of a single nucleotide insertion/deletion leading to a frameshift in the gene of strain A4 and position of translational fusion with the gus coding sequence in the rolA::gus gene, shown by arrows.

\section{Expression of the rolA::gus gene.}

As shown in Figure 2, the phenotype of the resulting rolA::gus plants was similar to that of a reference tobacco line (Carneiro and Vilaine 1993) expressing the native rolA gene, rolA(3), although the leaf wrinkling and stem shortening were less intense than in the rolA plants. When total RNA from rolA::gus and rolA plants was probed with a rolA-specific probe, a transcript of expected size was observed (Fig. 3A). Similarly, when a similar blot was probed with a GUSspecific probe a single band of expected size was observed (not shown). When electropherograms of total protein of rolA::gus plants and of plants expressing a $35 S$-gus gene were stained for GUS activity, the GUS-positive band in the rolA::gus plants was of higher molecular mass than that in 35S-gus plants (Fig. 3B).

When GUS activity was compared among five rolA::gus lines, there was a correlation between the level of this activity and the intensity of the phenotypic modifications (not shown). These results suggest that the rolA::GUS fusion protein is bifunctional, since plants expressing the rolA::gus gene displayed the typical rolA phenotype, and the RNA and protein observed corresponded to the size expected for the fusion.

\section{Subcellular localization of the rolA::GUS fusion protein.}

We compared the localization of GUS activity in several lines expressing the rolA::gus gene and one expressing a control 35S-gus gene. Figure 4A shows that, in 35S-gus plants, the highest GUS activity was found in the cytosol, with progressively lower GUS activities in the successively heavier fractions (cytosol > microsomes > mitochondria > chloroplasts $>$ nuclei). In contrast, the highest GUS activity was observed in the microsome fraction of all the rolA::gus lines tested. In particular, in RAG(6) plants, the microsomal GUS activity was five times that of the cytosolic activity. During

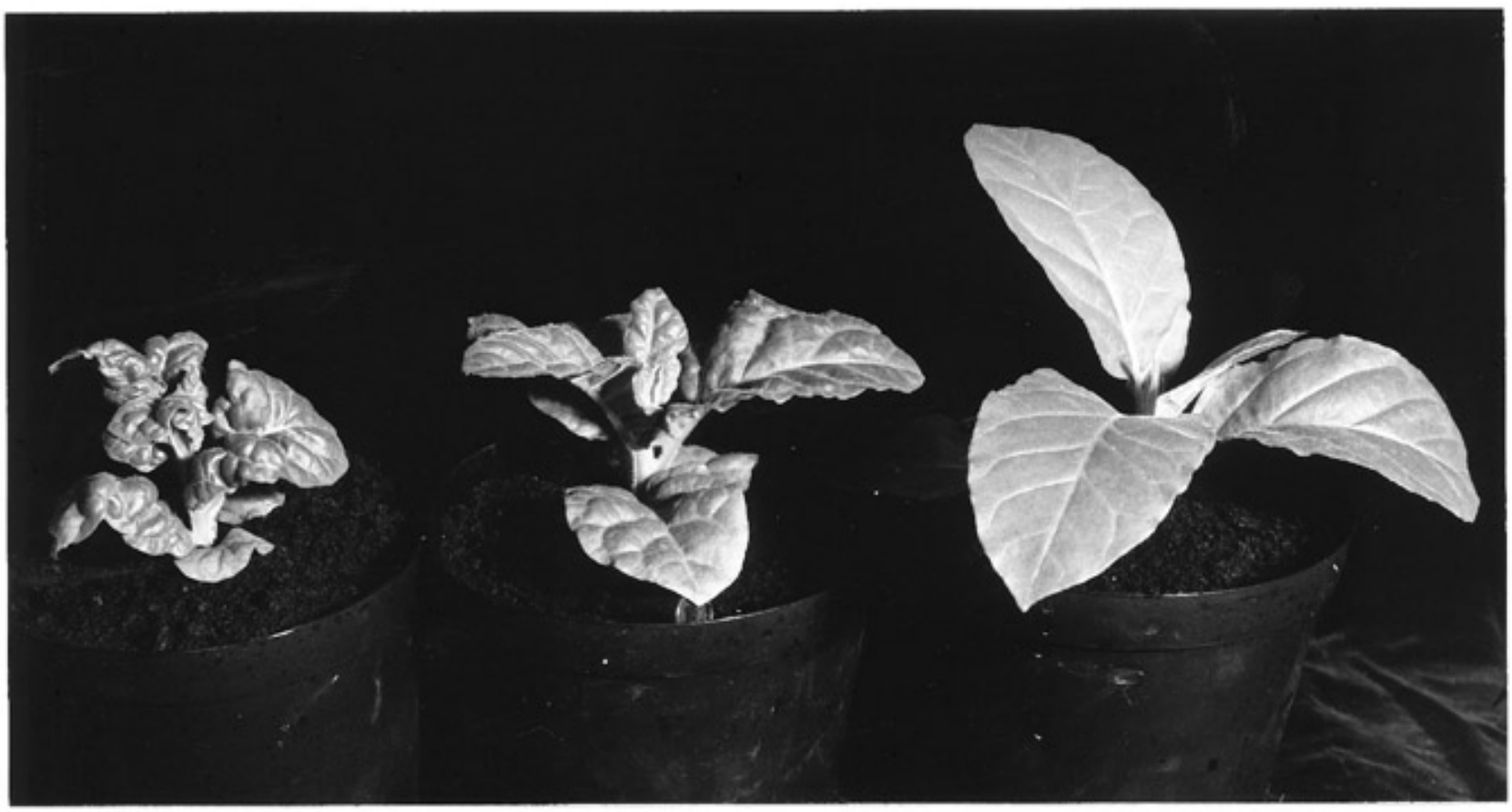

Fig. 2. Phenotype of tobacco plants expressing rolA and rolA::gus genes. Two-month-old homozygous plants of R2 generation are shown that express (left) native rolA gene (line rolA(3)), (center) rolA::gus gene (line RAG(3)), and (right) control, nontransformed tobacco. 
the steps of Percoll purification, the GUS activity of the nuclear fraction progressively decreased (not shown), which indicated that the nuclear GUS activity observed was due to contamination by non-nuclear material.

After fractionation of microsomes by phase partitioning, the different subcellular fractions of 35S-gus plants had similar levels of GUS activity; the membrane-associated activity could be due to cytosolic proteins entrapped within the vesicles, and/or to GUS protein weakly associated with the different membrane fractions (Fig. 4B). In contrast, all the rolA::gus lines had much higher GUS activity in the plasmalemma fraction than in the other fractions tested. For example, plasmalemma vesicles of RAG(1) plants had 15- and 42-fold higher GUS activity than the microsome and total protein fractions, respectively.

\section{DISCUSSION}

Transgenic tobacco plants expressing a rolA::gus translational fusion display the typical rolA phenotype. The results presented here clearly show that the rolA moiety of the rolA::GUS fusion protein strongly redirects GUS activity to the plasmalemma fraction, suggesting that the rolA protein itself is plasmalemma-associated. In contrast, it has been shown that another protein encoded by an A. rhizogenes gene, rolD, is cytosolic (Estruch et al. 1991). Thus, as we have shown that rolA::GUS is not nuclear, these results are not compatible with the hypothesis that rolA has a direct role in regulation of gene expression via protein-DNA interactions (Levesque et al. 1988). Since the rolA::GUS protein is plasmalemma-associated, the rolA sequence was further examined for potential structures that could be responsible for this localization. The N-terminal 25 residues are rich in hydrophobic amino acids (Fig. 1). However, since this hy-
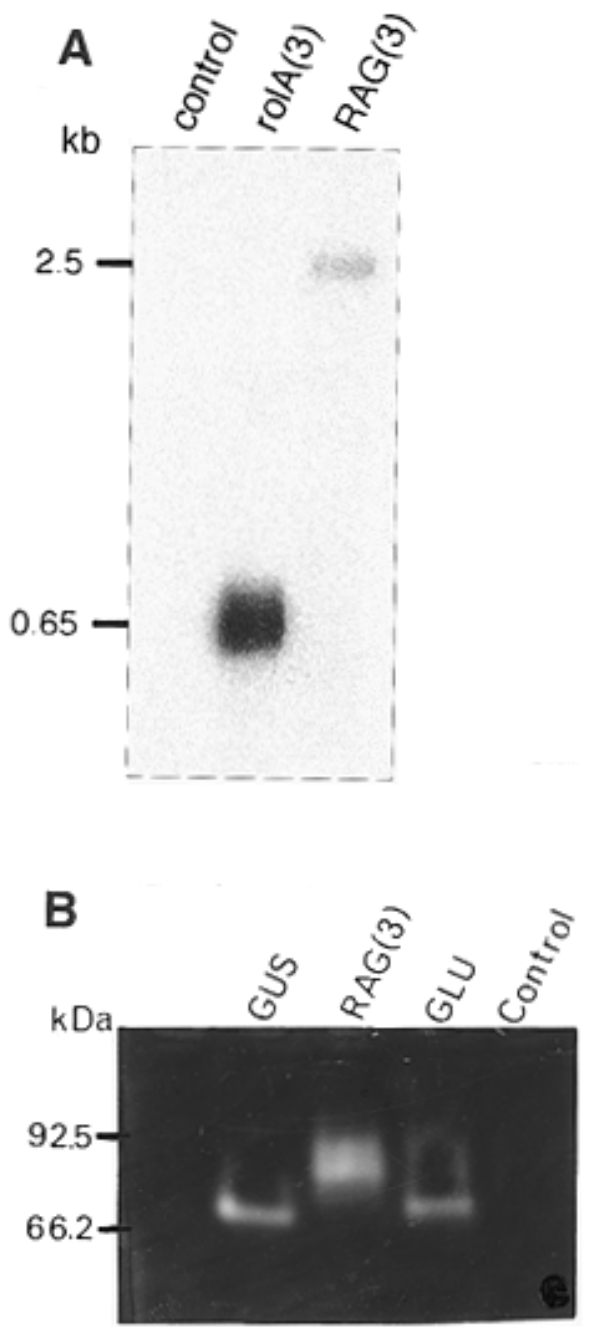

Fig. 3. Expression of rolA::gus gene in tobacco. A, Northern (RNA) blot of total leaf RNA ( $30 \mu \mathrm{g}$ per sample) probed with rolA coding sequence: control plant, rolA(3) plant, and RAG(3) plant. B, Visualization of $\beta$ glucuronidase (GUS) activity by polyacrylamide gel electrophoresis of total leaf protein (0.05 mg per sample): $35 \mathrm{~S}$-gus plant (GUS), RAG(3) plant, purified Escherichia coli GUS (Sigma, St. Quentin Fallavier, France) (GLU), and control plant (lane 4). Position of molecular mass markers is shown.
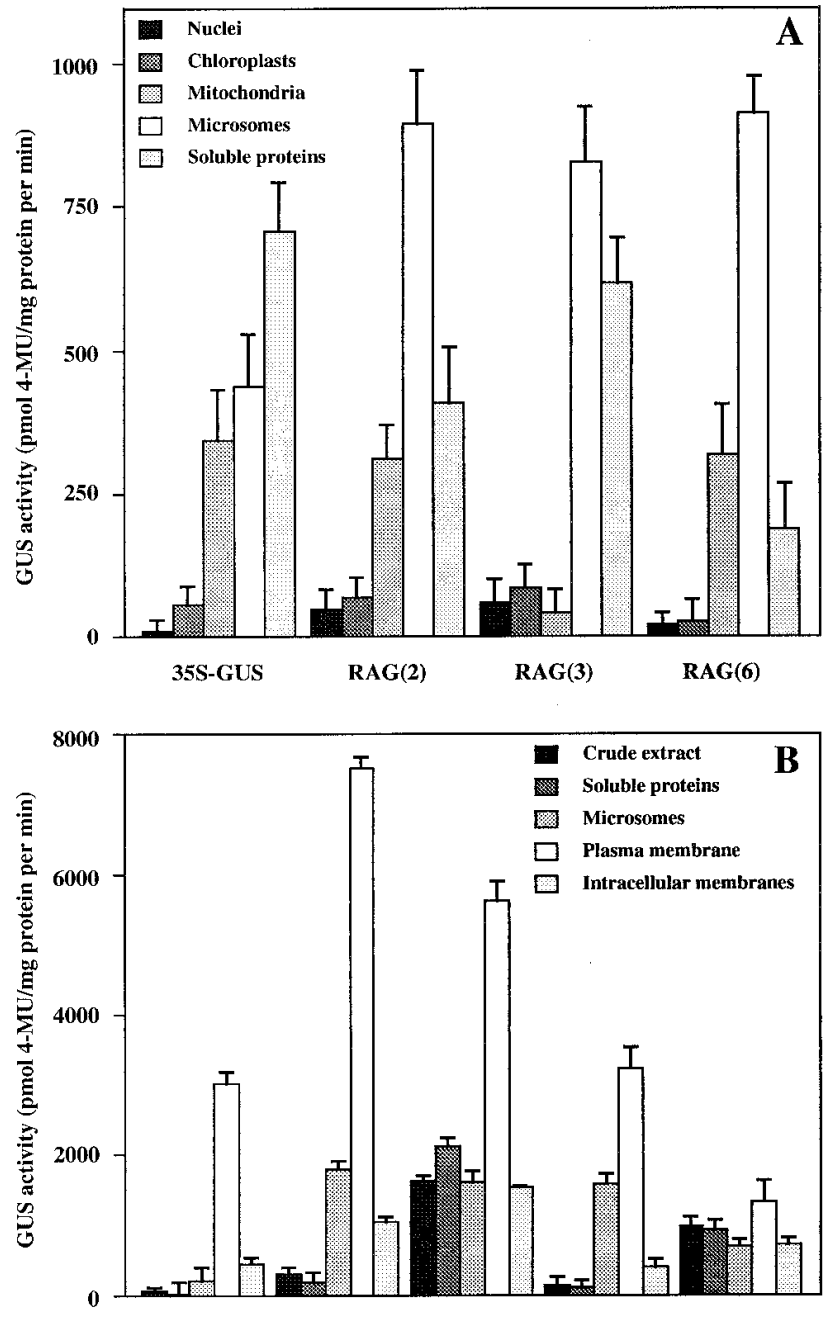

Fig. 4. $\beta$-Glucuronidase (GUS)-specific activity in subcellular fractions. GUS activity was determined in leaf extracts that had been separated into fractions enriched in (A) nuclei, chloroplasts, mitochondria, microsomes, and cytosol, and (B) total soluble protein, microsomes, plasmalemma, and intracellular microsomal membranes. Bars show mean values \pm SE obtained in three independent experiments. 
drophobic domain is interrupted by hydrophilic residues (Asn and Gln in the A4 rolA protein, with additional Asp and Glu in rolA of the other strains), it is unlikely that this domain could form a transmembrane segment. Likewise, the rolA proteins lack potential amphiphilic helices or sites of attachment of lipid anchors (not shown). The absence of what could clearly be transmembrane domains in the rolA protein is consistent with rolA::GUS being a non-integral, membrane-associated protein.

\section{MATERIALS AND METHODS}

\section{Sequence analysis of the rolA gene and its translation product.}

The sequences analyzed correspond to the rolA gene from A. rhizogenes strain A4 (accession numbers K03313 [Slightom et al. 1986] and X12579 [Sinkar et al. 1988]), strain 8196 (accession number M60490 [Hansen et al. 1991]), strain 2659 (accession number Z29365 [Serino et al. 1994]), and strain 1724 (accession number X64255 [Kiyokawa et al. 1994]). Sequence similarity was studied with various programs (BLAST, Pileup, Pretty, etc.) of the University of Wisconsin Genetics Computer Group (GCG) sequence analysis package (Devereux 1991).

\section{Construction of the rolA::gus gene and its transfer to the tobacco genome.}

A plasmid bearing the entire rolA gene in a $1.9-\mathrm{kb}$ EcoRI$N r u$ I fragment (pRA1) was cleaved with $N c o$ I and submitted to exonuclease Bal31 hydrolysis, after which fragments were rendered blunt-ended by Klenow polymerase treatment. The fragment containing the rolA gene was then isolated by XhoI hydrolysis, and cloned into pBS-KS ${ }^{+}$digested with SmaI and $X h o I$. A clone bearing the rolA open reading frame minus the last 15 nucleotides was selected. An XhoI-BamHI fragment containing the entire truncated gene was inserted into the plasmid pBRA1GUS (Carneiro and Vilaine 1993), replacing the XhoI-BamHI fragment bearing the rolA promoter, giving the plasmid prolAGUS, which was introduced into Agrobacterium tumefaciens strain GV3101(pMP90) (Koncz and Schell 1986) according to the method described by Zham et al. (1984). Transformed Nicotiana tabacum cv. Xanthi XHFD8 (Bourgin 1978) was obtained with the resulting Agrobacterium strain according to Horsch et al. (1985). The 35S-gus controls were obtained by transforming the same tobacco genotype with Agrobacterium bearing pBI121 (Jefferson 1987).

\section{Expression of the rolA::gus gene.}

Total RNA was extracted from leaves of 2-month-old, greenhouse-grown tobacco plants, according to the method described by Verwoed et al. (1989). Thirty micrograms of total RNA from each plant was submitted to electrophoresis in a denaturing agarose gel (Lehrach et al. 1977). After transfer to a nylon membrane (Pall Biodyne B) in 10× SSC (1× SSC is $0.15 \mathrm{M} \mathrm{NaCl}$ plus $0.015 \mathrm{M}$ sodium citrate), hybridization was performed at $50^{\circ} \mathrm{C}$ in the buffer of Church and Gilbert (1984). DNA corresponding precisely to the A4 rolA open reading frame was amplified from pRA1 by polymerase chain reaction, ${ }^{32} \mathrm{P}$-labeled by random priming, and used as probe (Feinberg and Vogelstein 1983).
Subcellular localization of the rolA::GUS fusion protein.

Differential centrifugation and Percoll purification were employed to separate homogenates into fractions enriched in chloroplasts, mitochondria, microsomes, and cytosol (Rembur et al. 1983). Intact nuclei were purified from mesophyll protoplasts according to Saxena et al. (1985). Plasmalemma was extracted from the 12,000 to $40,000 \mathrm{~g}$ of microsomal fraction by the aqueous, two-polymer, phase-partitioning method (Lundborg et al. 1981), with a final composition of $6.2 \%$ (wt/wt) Dextran T500 (Pharmacia, Orsay, France) and 6.2\% (wt/wt) polyethylene glycol 3350 (Sigma, St. Quentin Fallavier, France). Phase partitioning was conducted three times. Purity of the plasmalemma fraction obtained by phase partitioning was checked by measurements of ATPase activity at pH 6.5 (Perlin and Spanswick 1980). Measurements were carried out in the presence of various specific inhibitors according to Rembur et al. (1986). ATPase activity was inhibited $90 \%$ by $200 \mu \mathrm{M}$ orthovanadate, a specific inhibitor of plasmalemma ATPase. In contrast, the addition of $100 \mu \mathrm{M}$ molybdate (specific inhibitor of tonoplast ATPase) or $0.5 \mu \mathrm{g}$ of oligomycin (inhibitor of mitochondrial ATPase) per ml reduced activity only weakly (5 to $10 \%$ ). Absence of chlorophyll was shown according to Arnon (1949). These results show that there was very little contamination of the plasmalemma fraction.

GUS activity of fractions was determined with the fluorogenic substrate, 4-methylumbelliferyl glucuronide (MUG) according to Jefferson (1987). Protein content was determined by a modification of the Bradford method (Bio-Rad Laboratories, Ivry sur Seine, France), with bovine gamma globulin as standard. Data are expressed as picomoles of 4-MU liberated per minute per mg of protein.

Protein samples were analyzed on a $7.5 \%$ sodium dodecyl sulfate-polyacrylamide gel electrophoresis (SDS-PAGE) minigel (Laemmli 1970) after incubation at $40^{\circ} \mathrm{C}$ for $1 \mathrm{~min}$. Gels were washed in extraction buffer (Jefferson 1987), incubated in fresh buffer containing $0.5 \mathrm{mM}$ MUG for $20 \mathrm{~min}$, and rinsed in $0.2 \mathrm{M} \mathrm{Na}_{2} \mathrm{CO}_{3}$. Gels were photographed on a $312 \mathrm{~nm}$ transilluminator with Polaroid type 665 film.

\section{ACKNOWLEDGMENTS}

This research was supported in part by grant number 92.T.0355 from the French Ministry of Research.

\section{LITERATURE CITED}

Arnon, D. I. 1949. Copper enzymes in isolated chloroplasts. Polyphenoloxidase in Beta vulgaris. Plant Physiol. 24:1-15.

Ben-Hayyim, G., Martin-Tanguy, J., and Tepfer, D. 1996. Changing root and shoot architecture with the rolA gene from Agrobacterium rhizogenes: interactions with gibberellic acid and polyamine metabolism. Physiol. Plant. 96:237-243.

Bourgin, J.-P. 1978. Valine resistant plants from in vitro selected tobacco cells. Mol. Gen. Genet. 161:225-230.

Carneiro, M., and Vilaine, F. 1993. Differential expression of the rolA plant oncogene and its effect on tobacco development. Plant J. 3:785792.

Church, G. M., and Gilbert, W. 1984. Genomic sequencing. Proc. Natl. Acad. Sci. USA 81:1991-1995.

Dehio, C., Grossmann, K., Schell, J., and Schmülling, T. 1993. Phenotype and hormonal status of transgenic tobacco plants overexpressing the rolA gene of Agrobacterium rhizogenes T-DNA. Plant Mol. Biol. 23:1199-2110. 
Dehio, C., and Schell, J. 1993. Stable expression of a single-copy rolA gene in transgenic Arabidopsis thaliana plants allows an exhaustive mutagenic analysis of the transgene-associated phenotype. Mol. Gen. Genet. 241:359-366.

Devereux, J. 1991. The GCG sequence analysis software package, version 7.0. Genetics Computer Group, University Research Park, Madison, WI.

Estruch, J. J., Parets-Soler, A., Schmülling, T., and Spena, A. 1991. Cytosolic localization in transgenic plants of the rolC peptide from Agrobacterium rhizogenes. Plant Mol. Biol. 17:547-550.

Feinberg, A. P., and Vogelstein, B. 1983. A technique for radio-labelling DNA restriction endonuclease fragments to high specific activity. Anal. Biochem. 132:6-13.

Hansen G., Larribe, M., Vaubert, D., Tempé, J., Biermann, B. J., Montoya, A. L., Chilton, M.-D., and Brevet, J. 1991. Agrobacterium rhizogenes pRi8196 T-DNA: Mapping and DNA sequence of functions involved in mannopine synthesis and hairy root differentiation. Proc. Natl. Acad. Sci. USA 88:7763-7767.

Horsch, R. B., Fry, J. E., Hoffman, N. L., Eichholz D., Rogers, S. G., and Fraley, S. G. 1985. A simple and general method for transferring genes into plants. Science 227:1229-1231.

Jefferson, R. A. 1987. Assaying chimeric genes in plants: The GUS gene fusion system. Plant Mol. Biol. Rep. 5:387-405.

Kiyokawa, S., Kobayashi, K., Kikuchi, Y., Kamada, H., and Harada, H. 1994. Root-inducing region of mikimopine type Ri plasmid pRi1724. Plant Physiol. 104:801-802.

Koncz, C., and Schell, J. 1986. The promoter of TL-DNA gene 5 controls the tissue-specific expression of chimaeric genes carried by a novel type of Agrobacterium binary vector. Mol. Gen. Genet. 204: 383-396.

Laemmli, U. K. 1970. Cleavage of structural proteins during the assembly of the head of bacteriophage T4. Nature 227:680-685

Lehrach, H., Diamond-Wozney, J. M., and Boedtker, H. 1977. RNA molecular weight determination by gel electrophoresis under denaturing conditions, a critical reexamination. Biochemistry 16:4743-4751.

Levesque, H., Delepelaire, P., Rouzé, P., Slightom, J., and Tepfer, D. 1988. Common evolutionary origin of the central portions of the $\mathrm{Ri}$ TL-DNA of Agrobacterium rhizogenes and the Ti T-DNAs of Agrobacterium tumefaciens. Plant Mol. Biol. 11:731-744.

Lundborg, T., Widell, S., and Larsson, C. 1981. Distribution of ATPases in wheat root membranes separated by phase partition. Physiol. Plant. 52:89-95

Martin-Tanguy, J., Tepfer, D., Paynot, M., Burtin, D., Heisler, L., and Martin, C. 1990. Inverse relationship between polyamine levels and the degree of phenotypic alteration induced by the Ri TL-DNA from Agrobacterium rhizogenes. Plant Physiol. 92:912-918.

Maurel, C., Barbier-Brygoo, H., Spena, A., Tempé, J., and Guern, J. 1991. Single rol genes from Agrobacterium rhizogenes TL-DNA alter some of the cellular responses to auxin in Nicotiana tabacum. Plant Physiol. 97:212-216.
Perlin, D. S., and Spanswick R. M. 1980. Labeling and isolation of plasma membranes from corn leaf protoplasts. Plant Physiol. 65:10531057.

Prinsen, E., Chriqui, D., Vilaine, F., Tepfer, M., and Van Onckelen, H. 1994. Endogenous phytohormone levels in tobacco plants transformed with Agrobacterium rhizogenes pRi T-DNA genes. J. Plant Physiol. 144:80-85.

Raikhel, N. 1992. Nuclear targeting in plants. Plant Physiol. 100:16271632

Rembur, J., Landré, P., and Nougarède, A. 1983. Propriétés des activités ATPasiques mitochondriales et microsomales de l'épicotyle de Pois. Z. Pflanzenphysiol. 109:13-28.

Rembur, J., Landré, P., and Nougarède, A. 1986. Purification par séparation de phase et caractérisation du plasmalemme de l'épicotyle de pois. Biochem. Cell Biol. 64:448-455.

Saxena, P. K., Fowke, L. C., and King, J. 1985. An efficient procedure for isolation of nuclei from plant protoplasts. Protoplasma 128:184189

Schmülling, T., Schell, J., and Spena, A. 1988. Single genes from Agrobacterium rhizogenes influence plant development. EMBO J. 7:26292641.

Serino, G., Clerot, D., Brevet, J., Costantino, P., and Cardarelli, M. 1994. rol genes of Agrobacterium rhizogenes cucumopine strain: Sequence, effects and pattern of expression. Plant Mol. Biol. 26:415422.

Sinkar, V. P., Pythoud, F., White, F. F., Nester, E. W., and Gordon, M. P. 1988. The rolA locus of the Ri plasmid directs developmental abnormalities in transgenic plants. Genes Dev. 2:688-697.

Slightom, J. L., Durand-Tardif, M., Jouanin, L., and Tepfer, D. 1986 Nucleotide sequence analysis of TL-DNA of Agrobacterium rhizogenes agropine type plasmid: Identification of open reading frames. J. Biol. Chem. 261:108-121.

Tepfer, D. 1984. Transformation of several species of higher plants by Agrobacterium rhizogenes: Sexual transmission of the transformed genotype and phenotype. Cell 37:959-967.

Vansuyt, G., Vilaine, F., Tepfer, M., and Rossignol, M. 1992. rolA modulates the sensitivity to auxin of the proton translocation catalyzed by the plasma membrane $\mathrm{H}^{+}$-ATPase in transformed tobacco. FEBS Lett. 298:89-92.

Verwoed, T. C., Dekker, M. M., and Hoekema, A. 1989. A small-cale procedure for the rapid isolation of plant RNAs. Nucleic Acids Res. 6: 2362 .

White, F. F., Taylor, B. H., Huffman, G. A., Gordon, M. P., and Nester, E. W. 1985. Molecular and genetic analysis of the transferred DNA regions of the root-inducing plasmid of Agrobacterium rhizogenes. J. Bacteriol. 164:33-44.

Zham, P., Hohmeyer, C., and Geider, K. 1984. Site specific mutagenesis of the Ti plasmid by transformation by Agrobacterium tumefaciens with mutagenized T-DNA fragments cloned in E. coli plasmids. Mol. Gen. Genet. 194:188-194. 